\title{
Membrane tissue on the optic disc may cause macular schisis associated with a glaucomatous optic disc without optic disc pits
}

This article was published in the following Dove Press journal:

Clinical Ophthalmology

10 May 2013

Number of times this article has been viewed

\author{
Saori Takashina' \\ Wataru Saito' \\ Kousuke Noda' \\ Maki Katai ${ }^{2}$ \\ Susumu Ishida' \\ 'Department of Ophthalmology, \\ Hokkaido University Graduate School \\ of Medicine, ${ }^{2}$ Ophthalmology, Sapporo \\ Teishin Hospital, Sapporo, Japan
}

Correspondence: Wataru Saito

Department of Ophthalmology, Hokkaido University Graduate School of Medicine, Nishi 7, Kita I5, Kita-ku, Sapporo 060-8638, Japan

Tel +8I II 7065944

Fax+8I II 7065948

Email wsaito@med.hokudai.ac.jp

\begin{abstract}
This article reports a case of possible involvement of membrane tissue on the optic disc with macular schisis formation associated with glaucomatous optic disc without optic disc pits. A 78-year-old man presented with loss of central visual acuity of the left eye. He had a medical history of primary open-angle glaucoma. Visual acuity was 0.6 in his left eye, and funduscopy revealed macular schisis and a glaucomatous optic disc without optic disc pits. Optical coherence tomography (OCT) revealed membrane tissue on the optic disc and a tunnellike hyporeflective lesion connecting the schisis cavity and a site near the tissue, but no obvious optic disc pit. He underwent pars plana vitrectomy during which the membrane tissue on the disc and internal limiting membrane were removed. Posterior vitreous detachment was observed intraoperatively. Thereafter, the tunnel-like lesion observed on OCT was rapidly obscured and the macular schisis gradually reduced. Eighteen months after surgery, his visual acuity had improved to 0.9 with almost complete regression of the macular schisis. No optic disc pit was visualized after surgery. The changes in OCT findings described here suggest an etiology for macular schisis without optic disc pits in an eye with a glaucomatous optic disc with posterior vitreous detachment. A connection between the schisis cavity and the vitreous cavity may have appeared via the tunnel-like structure due to the membrane tissue exerting traction on the optic disc. Keywords: optical coherence tomography, pars plana vitrectomy, retinal schisis, glaucoma, optic disc pit maculopathy
\end{abstract}

\section{Introduction}

Macular schisis commonly occurs in eyes with pathological myopia or optic nerve head pits, ${ }^{1}$ and can cause deterioration of visual acuity. It has also been reported rarely in patients with glaucoma without optic disc pits. ${ }^{2-5}$ Although the pathogenesis in this context remains to be elucidated, some authors have inferred that a probable cause is the flow of vitreous fluids into the retina via a micro hole in the thin tissue of the optic disc triggered by ocular hypertension or vitreoretinal traction. ${ }^{2-4}$ In another patient with macular schisis and a normal optic disc without optic disc pits, a membrane-like structure was detected on the optic disc by optical coherence tomography (OCT), ${ }^{6}$ but no direct connection between the region bound by the membranous tissue and the schisis cavity was detected. Therefore, as far as we are aware, there are no previous reports that can verify the hypothesis.

We performed pars plana vitrectomy (PPV) with the removal of membrane tissue present on the optic disc in a patient with primary open-angle glaucoma and macular schisis without optic disc pits. The changes in the patient's OCT findings suggested that the membrane tissue on the optic disc was correlated with the pathogenesis of macular schisis formation in this patient. 


\section{Case report}

A 78-year-old man presented with central visual acuity loss in his left eye of 2 months' duration. He had been diagnosed with primary open-angle glaucoma of the oculus sinister (OS) 8 years earlier at another hospital, at which time treatment of the eye with latanoprost $0.005 \%$ was initiated. At that time, Humphrey threshold 30-2 perimetry showed decreased sensitivity in the Bjerrum areas, with a mean deviation value of $-3.40 \mathrm{~dB}$ in his left eye (Figure 1A). He had well-controlled diabetes mellitus treated by diet alone, and no significant medical family history.
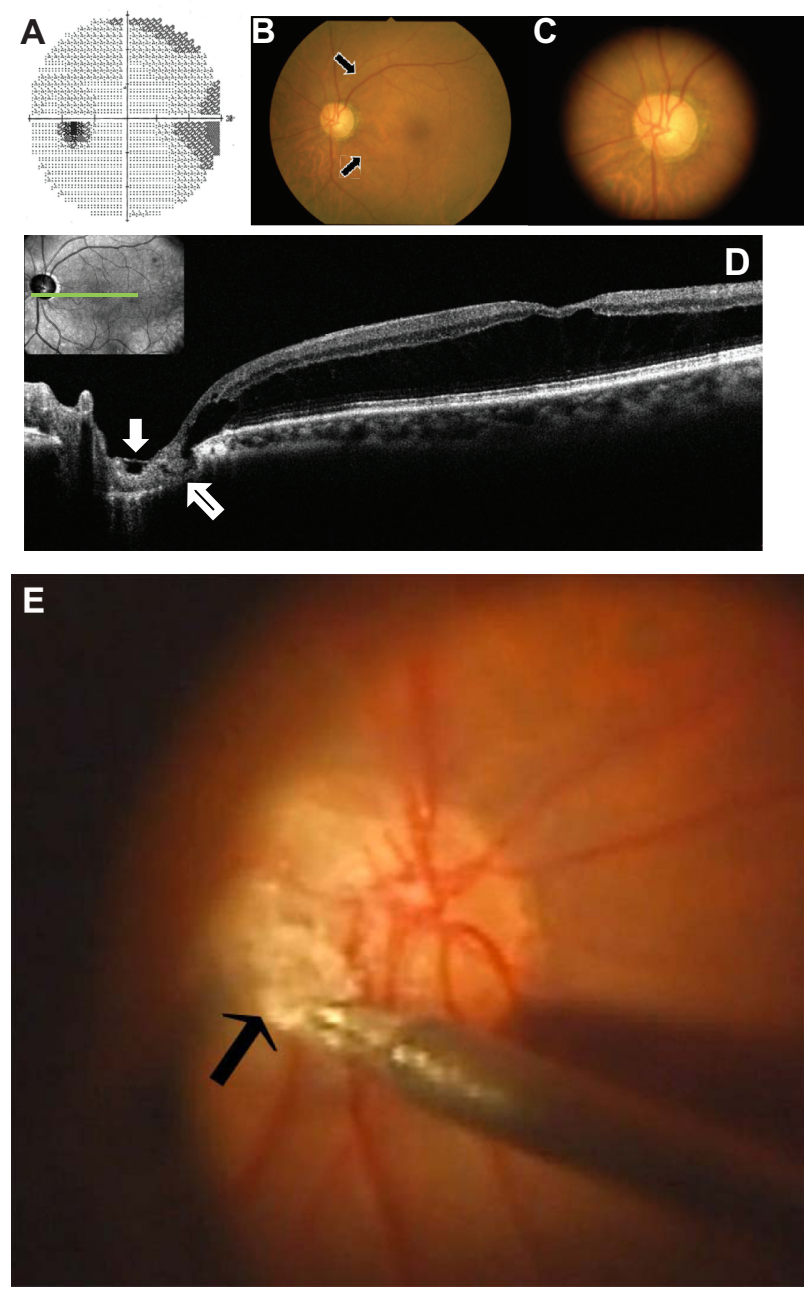

Figure I Photographs of the left eye before pars plana vitrectomy (A-D) and during surgery (E). (A) Humphrey threshold 30-2 perimetry was performed at another hospital, and the images from that procedure show decreased sensitivity in the Bjerrum's areas. (B and $\mathbf{C}$ ) Fundus photographs demonstrating retinal elevation with folds at the macula and optic nerve head cupping with nerve fiber layer defects (arrows) but no obvious optic disc pit. (D) Optical coherence tomography image showing retinal schisis extending from the optic disc to the macula, without retinal detachment, as well as membrane tissue with a sheet-like shape (white arrow) on the optic disc and a tunnel-like hyporeflective lesion (black arrow) directly connecting the retinal schisis and the 4 o'clock margin of the disc. (E) The membrane tissue on the optic disc grasped by forceps.
The patient's visual acuity measurements were 1.2 oculus dexter (OD) and $0.6 \mathrm{OS}$, with intraocular pressures of $15 \mathrm{mmHg}$ OD and $13 \mathrm{mmHg}$ OS at the first visit to our hospital. Slit-lamp examination revealed incipient cataract oculus uterque (OU), and gonioscopy revealed open-angle OU. In the right eye, there were no abnormal retinal findings except for a 0.8 cup on an optic nerve head. Funduscopic examination revealed retinal elevation with folds at the macula but no abnormal findings relating to the periphery OS (Figure 1B). The optic nerve head was large (the disc-tomacula distance to disc diameter ratio was 2.19) and had a 0.9 cup with nerve fiber layer defects (Figure 1B, arrows) but no obvious pit OS (Figure 1B and C). Fluorescein angiography showed no hyperfluorescence with leakages corresponding to the optic disc. Spectral domain OCT showed retinal schisis extending from the optic disc to the macula OS but no retinal detachment (Figure 1D). Membrane tissue with a sheet-like appearance on the optic nerve head (Figure 1D, white arrow) and a tunnel-like hyporeflective lesion directly connecting the schisis cavity and the 4 o'clock margin of the disc (Figure 1D, black arrow) were observed. Ocular axial length was $23.83 \mathrm{~mm}$ OD and $23.87 \mathrm{~mm}$ OS. Single-flash electroretinography findings were normal OU. Although optic disc pit maculopathy was suspected, no obvious pits were detected even when thorough OCT of multiple sections of the optic disc was undertaken. Thereafter, the patient's visual acuity increased to $1.0 \mathrm{OS}$.

After 18 months, the patient's visual acuity was found to have reduced to $0.7 \mathrm{OS}$, so he underwent PPV. Intraoperatively, posterior vitreous detachment (PVD) was observed. Internal limiting membrane in an area extending from the optic disc to the macula and the membrane tissue on the optic nerve head (Figure 1E, arrow) were removed. No gas tamponade was performed. No optic disc pit was observed during surgery. The tunnel-like hyporeflective lesion on OCT was obscured from the early postoperative stage (Figure 2A and B). No optic disc pits were observed on OCT after surgery. Twelve months after the operation, the retinal schisis was observed to have gradually reduced, and the tunnel-like lesion had completely resolved (Figure 2C, arrow), although the mean deviation value on Humphrey perimetry had worsened to $-4.70 \mathrm{~dB}$. Eighteen months after surgery, the patient's vision was found to have improved to 0.9 and the retinal schisis to have almost completely resolved (Figure 2D and E).

\section{Discussion}

Herein, we report the case of a patient with macular schisis and a glaucomatous optic disc without optic disc pits. 

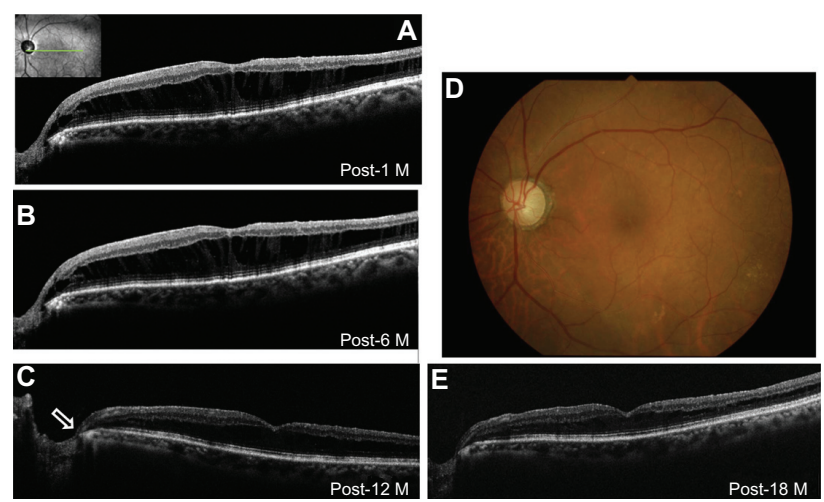

Figure 2 Photographs of the left eye after vitrectomy. (A) Optical coherence tomography (OCT) image showing that the tunnel-like lesion in the optic disc was obscured I month after surgery and (B) retinal schisis was reduced 6 months after surgery. (C) Twelve months after surgery, OCT revealed that the tunnel-like hyporeflective lesion (black arrow) had resolved and retinal schisis had decreased further. (D) Fundus photograph taken 18 months after surgery showing the decrease in retinal elevation at the macula and no optic disc pit. (E) OCT image showing normalization of central retinal thickness with almost complete resolution of retinal schisis.

Abbreviation: M, month/s.

Membrane tissue on the patient's optic disc and a tunnel-like hyporeflective lesion directly connecting the retinal schisis and the optic nerve head were detected. The tunnel-like lesion was rapidly obscured after PPV, during which the membrane tissue was removed. Macular schisis gradually resolved and visual acuity improved.

The mechanisms responsible for the retinal schisis associated with optic disc pits and a glaucomatous disc remain to be clarified and surgical approaches to repair retinal schisis have not been fully established. Optic disc pit maculopathy appears to have a predominant predilection for young people without PVD. ${ }^{1,7,8}$ In $62.5 \%$ (10/16) of eyes with maculopathy, vitreomacular traction extending from the optic nerve head to the macula was detected on OCT preoperatively. ${ }^{7}$ Interestingly, the presence of dense vitreous strands adhering to optic disc pits, resembling the membrane tissue in our present case, has been previously documented. ${ }^{7,9}$ In $87.5 \%$ (7/8) of eyes with optic disc pit maculopathy without PVD, maculopathy resolved following PPV with induction of PVD at the optic disc, without laser photocoagulation or gas tamponade. ${ }^{8}$ In two eyes with glaucoma developing retinal schisis without PVD, retinal schisis was reduced after PPV with intraoperative PVD formation. ${ }^{3}$ Based on these observations, it is suggested that traction of the posterior hyaloid to the optic disc plays a role in the onset of retinal schisis associated with optic disc pits or a glaucomatous disc without PVD. ${ }^{1,8,10}$

However, in the case discussed here, PVD was observed preoperatively. Therefore, multiple scans for the optic disc with spectral domain OCT were performed to examine the etiology causing retinal schisis, and the membrane tissue on the disc and the communication directly connecting the schisis cavity and the 4 o'clock margin of the disc were depicted. The membrane tissue adhered to the rim-defective site on the disc. The tunnel-like communication was rapidly obscured after PPV with removal of the tissues without gas tamponade, resulting in resolution of the macular schisis. The membrane tissue per se was likely to be the only medium of traction force to the optic disc, as well as epiretinal membranes after PVD. Thus, we speculate that contraction of the membrane tissue on the vulnerable optic disc caused a tiny cleft, which in turn led to communication between the vitreous cavity and the retinal parenchyma. The shape of the tunnel observed on OCT suggests that the fluids that flowed into the retina originated from the vitreous but not cerebrospinal fluids. OCT failed to detect an apparent break within the optic disc in this case, suggesting that the opening cleft was minute enough to close without gas tamponade if only traction was released by removing the membrane tissue. This speculation is compatible with and supported by the observation that retinal schisis has been reported to resolve following PPV without gas tamponade in cases with optic disc pit maculopathy. ${ }^{8}$

To the best of our knowledge, this is the first report to demonstrate a possible cause-effect relationship between membrane tissue on the optic disc and macular schisis associated with a glaucomatous optic disc in an eye without optic disc pits. Our findings indicate that in such cases the membrane tissue should be removed from the optic disc and that gas tamponade is not necessary during PPV.

In contrast to our hypothesis, among eight eyes with optic disc pits or colobomas, $60 \%(3 / 5)$ of eyes without maculopathy had membrane tissues traversing the optic disc, whereas $100 \%(3 / 3)$ of eyes with maculopathy did not, ${ }^{11}$ suggesting that tissues on the optic disc may prevent the influx of vitreous fluids into the retina and protect from retinal schisis formation. However, all three eyes with maculopathy belonged to young patients without PVD, suggesting an association of the posterior hyaloid adhering to the optic disc with the pathogenesis of maculopathy. In addition, of four eyes with optic disc pit maculopathy, two without preoperative PVD showed resolution of maculopathy after PPV with induction of PVD, whereas the other two eyes with no resolution belonged to elderly patients having PVD before surgey. ${ }^{10}$ Taken together, this suggests that in young patients without PVD, the posterior hyaloid adhering to pits may exert traction on the disc and cause maculopathy, 
whereas, in elderly patients with PVD such as ours, remnant tissue contraction on the disc is likely to be a driving force similar to the posterior hyaloid or epiretinal membranes.

Our patient underwent removal of the adherent membrane tissue together with internal limiting membrane (ILM) peeling, which may have played an additive role in the resolution of his maculopathy. However, in another study, ILM peeling was not performed during PPV with induction of PVD in $85.7 \%(6 / 7)$ of eyes with optic disc pit maculopathy that successfully achieved anatomical improvement. ${ }^{8}$ These results suggest that ILM makes less of a contribution to maculopathy development as a traction force than the posterior hyaloid or remnant tissues attached to the optic nerve head.

In the present case, the visual field of the left eye deteriorated after PPV (from $-3.40 \mathrm{~dB}$ to $-4.70 \mathrm{~dB}$ ). We cannot exclude the possibility that the removal of the membrane tissues might have caused damage to the optic nerve head because the tissue adhered to the thinnest (ie, rim-defective) region on the disc, although it is difficult to determine whether the visual field aggravation was caused by the surgical stress or the progression of glaucoma. When vitreous surgeons remove the membrane tissues on the disc, as in this case, a risk-benefit assessment is required.

It is of great importance to differentiate between tunnellike hyporeflective lesions (Figure 1D, black arrow) and OCT scan artifacts such as obscure images with hyporeflection due to involuntary subtle eye movements during fixation and a tilt of materials. To avoid these artifacts, we enhanced the quality of the images by averaging multiple (ten) spectral domain OCT scans. Other factors that may lead to misleading hyporeflection include a shadowing effect caused by blocking a measuring beam, resulting in a linear shape extending backwards and vertically. In our case, we could rule out the possibility of the shadowing artifact because the tunnel-like lesion was apparently U-shaped.

Recently, focal lamina cribrosa defects were demonstrated using enhanced depth imaging OCT in 75.6\% (34/45) of eyes with glaucoma. Of the eyes with defects, $24.4 \%(11 / 45)$ involved an acquired pit of the optic nerve. ${ }^{12}$ Although in the present case it was unknown whether there were focal lamina cribrosa defects because enhanced depth imaging OCT was not undertaken, no acquired pit was detected fundoscopically or with spectral domain OCT, even after surgery with membrane removal.

\section{Conclusion}

In a patient with macular schisis and a glaucomatous optic disc without optic disc pits, OCT revealed membrane tissue on the optic disc and a tunnel-like hyporeflective lesion connecting the schisis cavity and a site near the tissue. After PPV with removal of membrane tissue, on OCT, the macular schisis was seen to have gradually resolved following resolution of the tunnel lesion. These results suggest that membrane tissues on the optic disc play a role in the pathogenesis of the macular schisis associated with glaucomatous eyes without adhesion between the posterior vitreous and the optic nerve head.

\section{Disclosure}

The authors declare no conflicts of interest in this work.

\section{References}

1. Georgalas I, Ladas I, Georgopoulos G, Petrou P. Optic disc pit: a review. Graefes Arch Clin Exp Ophthalmol. 2011;249(8):1113-1122.

2. Hollander DA, Barricks ME, Duncan JL, Irvine AR. Macular schisis detachment associated with angle-closure glaucoma. Arch Ophthalmol. 2005;123(2):270-272.

3. Zumbro DS, Jampol LM, Folk JC, Olivier MM, Anderson-Nelson S. Macular schisis and detachment associated with presumed acquired enlarged optic nerve head cups. Am J Ophthalmol. 2007;144(1): $70-74$.

4. Kahook MY, Noecker RJ, Ishikawa H, et al. Peripapillary schisis in glaucoma patients with narrow angles and increased intraocular pressure. Am J Ophthalmol. 2007;143(4):697-699.

5. Zhao M, Li X. Macular retinoschisis associated with normal tension glaucoma. Graefes Arch Clin Exp Ophthalmol. 2011;249(8): $1255-1258$

6. Spaide RF, Costa DL, Huang SJ. Macular schisis in a patient without an optic disk pit optical coherence tomographic findings. Retina. 2003; 23(2):238-240.

7. Theodossiadis PG, Grigoropoulos VG, Emfietzoglou J, Theodossiadis GP. Vitreous findings in optic disc pit maculopathy based on optical coherence tomography. Graefes Arch Clin Exp Ophthalmol. 2007;245(9):1311-1318.

8. Hirakata A, Inoue M, Hiraoka T, McCuen BW 2nd. Vitrectomy without laser treatment or gas tamponade for macular detachment associated with an optic disc pit. Ophthalmology. 2012;119(4):810-818.

9. Hirakata A, Hida T, Wakabayashi T, Fukuda M. Unusual posterior hyaloid strand in a young child with optic disc pit maculopathy: intraoperative and histopathological findings. Jpn J Ophthalmol. 2005; 49(3):264-266.

10. Haruta M, Kamada R, Umeno Y, Yamakawa R. Vitrectomy for optic disc pit-associated maculopathy with or without preoperative posterior vitreous detachment. Clin Ophthalmol. 2012;6:1361-1364.

11. Doyle E, Trivedi D, Good P, Scott RA, Kirkby GR. High-resolution optical coherence tomography demonstration of membranes spanning optic disc pits and colobomas. Br J Ophthalmol. 2009;93(3):360-365.

12. Kiumehr S, Park SC, Syril D, et al. In vivo evaluation of focal lamina cribrosa defects in glaucoma. Arch Ophthalmol. 2012;130(5):552-559. 
Clinical Ophthalmology

\section{Publish your work in this journal}

Clinical Ophthalmology is an international, peer-reviewed journal covering all subspecialties within ophthalmology. Key topics include: Optometry; Visual science; Pharmacology and drug therapy in eye diseases; Basic Sciences; Primary and Secondary eye care; Patien Safety and Quality of Care Improvements. This journal is indexed on

PubMed Central and CAS, and is the official journal of The Society of Clinical Ophthalmology (SCO). The manuscript management system is completely online and includes a very quick and fair peer-review system, which is all easy to use. Visit http://www.dovepress.com/ testimonials.php to read real quotes from published authors. 